\title{
Study of glucose concentration influence on blood optical properties in $\mathrm{THz}$ frequency range
}

\author{
S. I. Gusev ${ }^{1}$, P. S. Demchenko ${ }^{1}$, E. A. Litvinov ${ }^{1}$, \\ O. P. Cherkasova ${ }^{2,3}$, I. V. Meglinski ${ }^{4}$, M. K. Khodzitsky ${ }^{1}$ \\ ${ }^{1}$ Terahertz Biomedicine laboratory, ITMO University, \\ Kronverkskiy Ave, 49, St. Petersburg, 197101, Russia \\ ${ }^{2}$ Biophysics laboratory, Institute of Laser Physics of SB RAS, \\ Ac. Lavrentiev Ave, 13, Novosibirsk, 630090, Russia \\ ${ }^{3}$ Novosibirsk State Technical University, \\ K. Marksa Ave, 20, Novosibirsk, 630073, Russia \\ ${ }^{4}$ University of Oulu, P.O. Box 4500, FI-90015, Oulu, Finland \\ mail@gusev-spb.ru, khodzitskiy@yandex.ru
}

\begin{abstract}
The optical properties of whole human blood with the different glucose level were studied by terahertz time-domain spectroscopy at frequencies ranging from $0.3-0.5 \mathrm{THz}$. The increasing of refractive index of blood at the glucose level growth was shown for series of experiments. The dispersion of complex refractive index of human nails was obtained. Based on these data, the non-invasive glucose measuring technique was proposed which utilizes the reflection of the THz pulse from nail plate/nail bed interface.
\end{abstract}

Keywords: spectroscopy, THz frequency range, blood, glucose, diabetes.

Received: 7 April 2018

Revised: 14 May 2018

\section{Introduction}

Currently, terahertz time-domain spectroscopy (THz TDS) and imaging techniques are applied in various fields such as astronomy, security screening, communications, genetic engineering, pharmaceutical quality control, medical imaging and biomedical engineering [1-4].

Several unique features make THz-based technology very suitable for medical applications. The main feature of $\mathrm{THz}$ spectroscopy which is really important for biophotonics is that the characteristic energies of molecules rotational and vibrational motions lay in $\mathrm{THz}$ frequency region, so many chemical and biological molecules can be identified by their characteristic resonant peaks [5]. Also THz radiation is very sensitive to water [6], so it could provide a great contrast of samples with different water content. In addition, $\mathrm{THz}$ radiation is non-ionizing due to it has very low photon energy. As a result, it might be safely applied to in vivo diagnostics. This fact is a significant advantage for biological structure invetigation, because there does not appear to be any tissue damage during the procedure $[7,8]$.

Delayed diagnostics of diabetes mellitus as well as insufficient control on patient are the general reasons of complications, which lead to invalidation and higher mortality. It should to be noted that a large number of patients have macrovascular complications due to unnormal blood glucose level: hyperglycemia, hypoglycemia, variability of glycemia. Statistics shows that growth of glycated hemoglobin (HbA1c) index from $6 \%$ to $9 \%$ leads to a four-fold increased risk of microvascular complications and twice the risk of myocardial infarction [9]. Hypoglycemia (even in subclinical situations) is associated with a $2-2.5$-fold increased risk of mortality from cardiovascular complications and a four-fold increase in the number of cardiovascular cases in [10]. Variability of glycemia is another risk factor, as variability of glucose level by more than $60 \mathrm{mg} / \mathrm{dl}$ is a predictor of coronary atherosclerosis (2.6-fold increased risk) even more accurate than HbA1c [11]. In addition, glycemia variability on an empty stomach is an independent predictor of cardiovascular lethal cases for senior patients with diabetes mellitus type 2 [12], urgent patients [13] and patients with myocardial infarction [14].

The present clinical methods of glucose level sensing include the following: opto-chemical (Accu-Chek Active) and electro-chemical (Bayer Contour TS, Abbott Freestyle, OneTouch Select) glucometers, continuous glucose monitoring sensors (Medtronic MiniMed, Freestyle Libre), electro-chemistry for labs (EKF Biosen C), all of which require fresh blood sampling. At the same time, there is still lack of non-invasive methods of glucose measuring: some are out of production due to drawbacks [15], while other are only at experimental stages [16,17], 
or slowly coming to market $[18,19]$. Some papers show great skepticism about the possibility of completely non-invasive glucose measuring techniques [20].

Despite these facts and opinions, this paper is part of a series of works [21-23], the goal of which is noninvasive glucose measuring technique which can use benefits of $\mathrm{THz}$ radiation for biomedical diagnostics purposes.

This work presents both transmission and reflection spectroscopic methods in $\mathrm{THz}$ frequency range to analyze glucose level in blood samples. The transmission mode is simpler to maintain and it was used to make in vitro analysis of blood and nail samples. Collected data was used for modeling of in vivo non-invasive experiment in CST Microwave Studio software.

\section{Experimental setup}

The optical properties of blood samples were experimentally studied at frequencies ranging from $0.3-0.5 \mathrm{THz}$ using THz TDS in transmission mode [24]. The scheme of the setup is shown in Fig. 1.

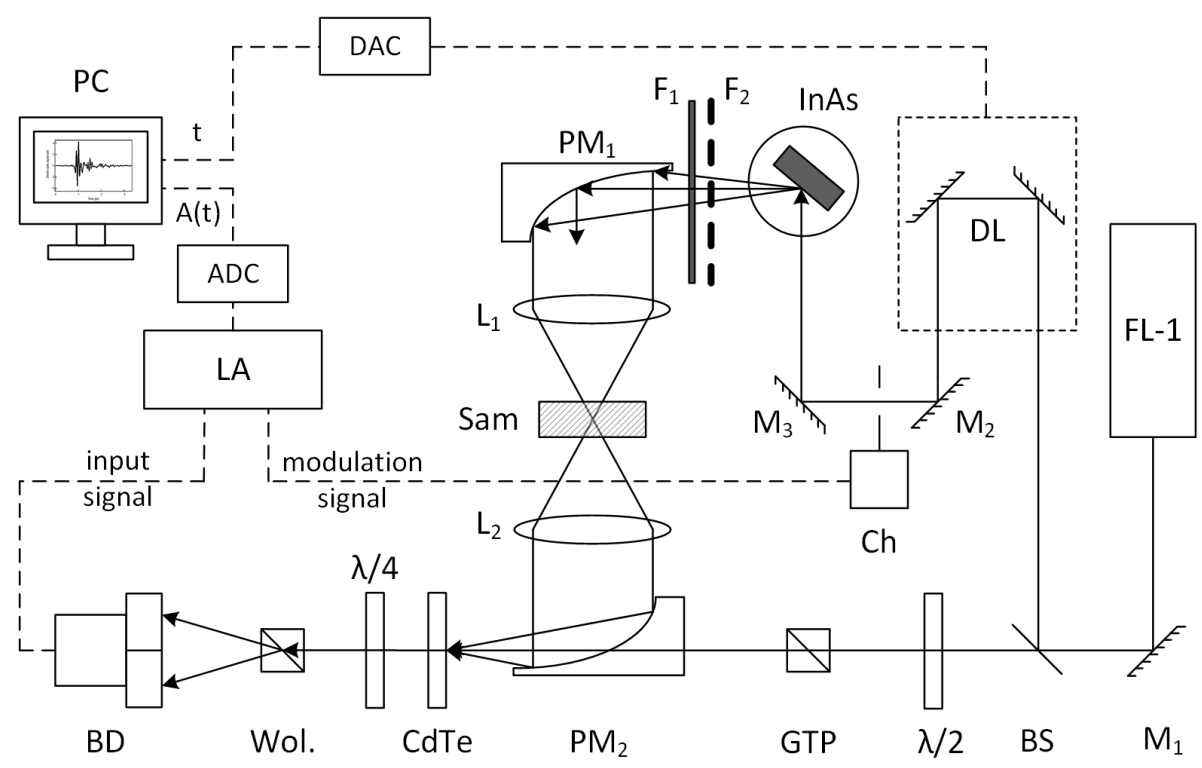

FIG. 1. Experimental THz TDS setup in transmission mode (FL-1 - femtosecond laser based on potassium-yttrium tungstate crystal activated with ytterbium ( $\mathrm{Yb}$ : KYW), generating femtosecond pulses; F1,2 - a set of teflon filters for IR wavelength range cutting off, BS - beamsplitter, DL optical delay line, M1,2,3 - mirrors, Sam - investigated sample, Wol. - Wollaston prism, CdTe electro-optical cadmium-telluric crystal, BD - balanced detector, LA - lock-in amplifier, PC personal computer, GTP - Glan-Taylor prism, PM1,2 - parabolic mirrors, Ch - chopper, DAC digital to analog converter, ADC - analog to digital converter)

Broadband pulsed THz radiation is generated by means of an InAs semiconductor in a $2.0 \mathrm{~T}$ magnetic field by irradiating it with femtosecond pulses of an $\mathrm{Yb}$ : KYW laser (wavelength of $1040 \mathrm{~nm}$, at a pulse duration of $120 \mathrm{fs}$, the pulse repetition frequency of $75 \mathrm{MHz}$, the power of $1 \mathrm{~W}$ ). THz radiation has the following output characteristics: the pulse duration of $2.7 \mathrm{ps}$, the main power is concentrated at the frequency range from 0.3 to $0.5 \mathrm{THz}$ with an average power up to $30 \mu \mathrm{W}$, which was measured by a Golay cell. The typical transmission spectrum of air for this THz TDS is shown in Fig. 2 (Spectral resolution is $1 \mathrm{GHz}$ ). THz radiation passes through a teflon filter (which omits wavelengths shorter than $50 \mu \mathrm{m}$ ). After that, the $\mathrm{THz}$ radiation passes through the sample fixed in a focal plane perpendicular to the beam. The existence of the THz field changes the birefringence of the electroopical crystal, i.e. causing the refractive index difference for polarizations along different axes of the crystal. The electric field-induced birefringence changes the probe beam's polarization [25]. As a result, the THz pulse induces birefringence of the probe beam in the crystal due to the electrooptical effect. The birefringence magnitude is directly proportional to the amplitude of terahertz radiation electric field in the time point $E(t)$. These data are required to calculate $E(\omega)$ using the Fourier transform. 


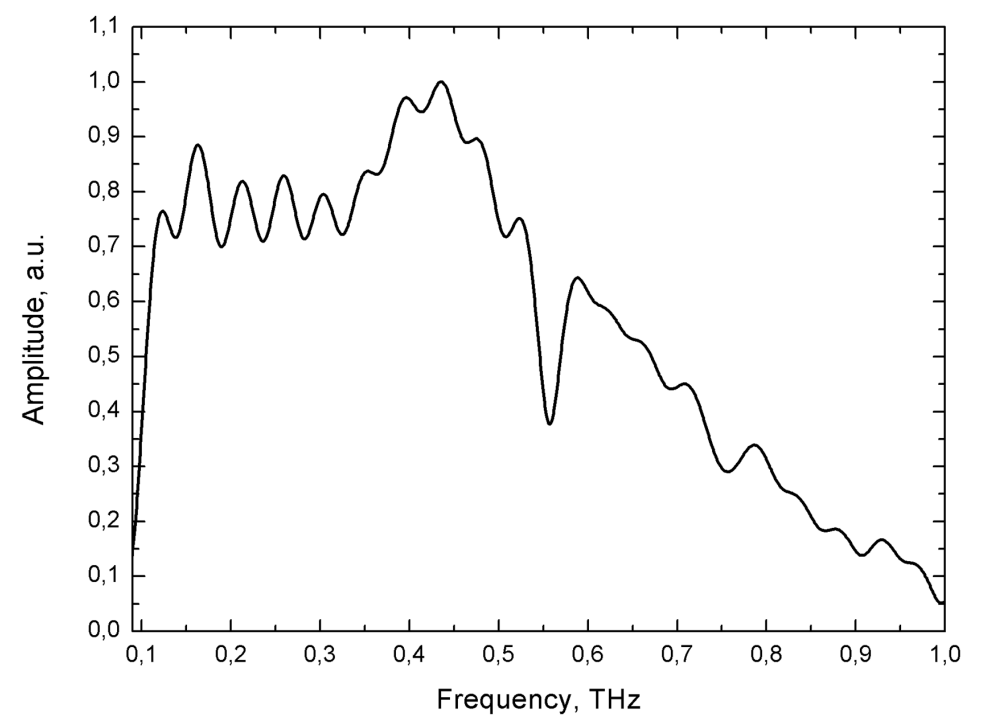

FIG. 2. Transmission spectrum of air

\section{Sample preparation}

In the experiment, 8 blood samples were used, each with a different glucose concentration. The samples under study were obtained from the same person over a short time period (about 2 hours). The patient had no significant diseases other than diabetes mellitus. The patient had an increased levels of: glycated hemoglobin (7,9\%); LD cholesterol $(4.60 \mathrm{mmol} / \mathrm{l})$; uric acid $(9,1 \mathrm{mmol} / \mathrm{l})$. Other blood components levels were normal. This fact helped us to provide the stable concentration of blood components (excluding glucose concentration) to avoid multiple dependences within the time of experiment. The study was performed in accordance with Good Clinical Practice (GCP) and with the 1964 Helsinki declaration and its later amendments. All measurements were performed with assistance and control of the staff of the Institute of Endocrinology of Federal Almazov North-West Medical Research Center (St.Petersburg, Russia).

The first step of sample preparation was increasing glucose level of the diabetic patient up to hyperglycemia level. It is quite a fast process so samples were obtained during the time of decreasing glucose level after insulin injection. This experiment contained samples with the following glucose concentrations shown in Table 1. The glucose concentrations in the blood were measured twice per a sample using Abbott Freestyle Optium [26] glucometer. This instrument shows concentration in values of mmol/l standard, which can be converted to $\mathrm{mg} / \mathrm{dl}$ by multiplication by 18 . This model of electro-chemical glucometer model is widely used in clinical research [27]. The glucose concentration measurement and THz test happened simultaneously.

The blood drops were located inside a special polymethyl methacrylate (PMMA) container (Figs. 3,4). In the middle this container was a recess with a depth of $75 \mu \mathrm{m}$. This type of blood holder provides keeping stable thickness of a sample and protects biological fluid from drying.

TABLE 1. The list of the glucose concentrations of samples used in experiment.

\begin{tabular}{|c|c|c|}
\hline Sample \# & $\mathrm{mmol} / \mathrm{l}$ & $\mathrm{mg} / \mathrm{dl}$ \\
\hline 1 & 3.0 & 54.0 \\
\hline 2 & 3.8 & 68.4 \\
\hline 3 & 6.2 & 111.6 \\
\hline 4 & 9.2 & 165.6 \\
\hline 5 & 11.0 & 198.0 \\
\hline 6 & 14.9 & 268.2 \\
\hline 7 & 18.0 & 324.0 \\
\hline 8 & 19.0 & 342.0 \\
\hline
\end{tabular}



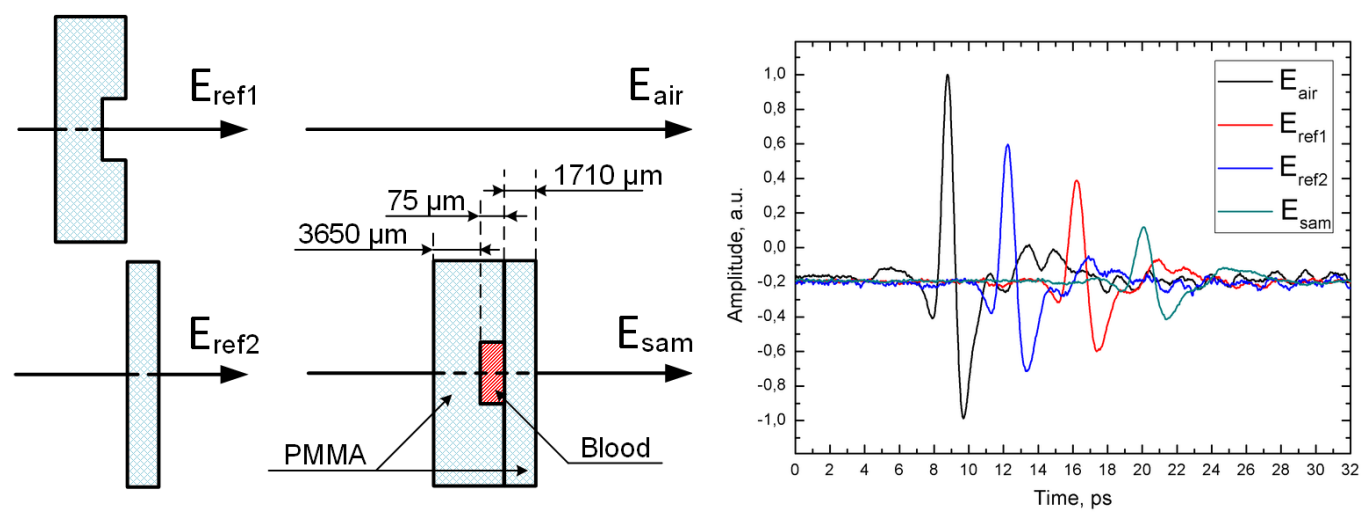

FIG. 3. The scheme of sample preparation and experimental $\mathrm{THz}$ waveforms

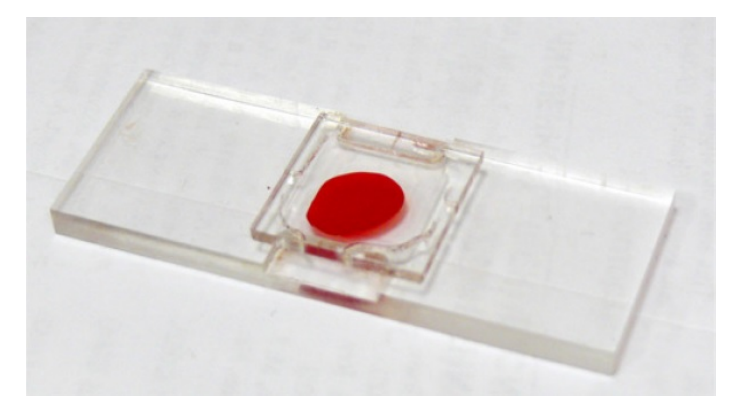

FIG. 4. Polymethyl methacrylate (PMMA) container with blood

\section{Data acquisition}

For each sample, a time-amplitude transmission waveform was taken 100 times and averaged for each timepoint. In addition, transmission waveforms of air, bottom and top parts of container were measured. All the acquired waveforms were converted to the frequency domain $\hat{E}(\omega)$ using Fourier transform. Then, the THz electric field is:

$$
\hat{E}(\omega)=\frac{1}{\sqrt{2 \pi}} \int_{-\infty}^{+\infty} f(t) \exp (-i \omega t) d t=E_{0}(\omega) \exp (-i \varphi(\omega)),
$$

where $f(t)$ is the time-amplitude waveform, $\omega$ is the angular frequency, $E_{0}(\omega)$ is the amplitude data, $\varphi(\omega)$ is the phase data.

At the first step, $E_{r e f 1}(t), E_{r e f 2}(t)$, THz waveforms were used to obtain the phase delay dispersions for the bottom part and the top part of container, $\Delta \varphi_{1}(f)$ and $\Delta \varphi_{2}(f)$ correspondingly, where $\Delta \varphi_{1}(f)=\varphi_{\text {ref } 1}(f)-$ $\varphi_{\text {air }}(f), \Delta \varphi_{2}(f)=\varphi_{\text {ref } 2}(f)-\varphi_{\text {air }}(f)$. At the second step $E_{\text {sam }}(t) \mathrm{THz}$ waveform was used to obtain the phase dispersion for the container with blood $\varphi_{\text {sam }}(f)$. Then the phase delay dispersion for blood was calculated as: $\Delta \varphi_{\text {blood }}(f)=\varphi_{\text {sam }}(f)-\varphi_{\text {ref }}(f)$, where $\varphi_{\text {ref }}(f)=\varphi_{\text {air }}(f)+\Delta \varphi_{1}(f)+\Delta \varphi_{2}(f)$. The phase delay dispersion for blood $\Delta \varphi_{\text {blood }}(f)$ was used to calculate complex refractive index and complex permittivity.

As a result, the refractive index $n$ can be calculated for a sample inside the container. The real part of the blood refractive index $n_{\text {real blood }}$ is calculated as:

$$
n_{\text {real blood }}(f)=1+\frac{c\left(\varphi_{\text {sam }}(f)-\varphi_{\text {ref }}(f)\right)}{2 \pi f d_{\text {blood }}},
$$

where $c$ is the speed of light in vacuum, $d_{\text {blood }}$ is the thickness of blood layer, $f$ is the frequency, ref and sam indices mean values attitude to reference and sample signals correspondingly.

The absorption coefficients $\alpha$ are calculated using the electric field amplitude data:

$$
d_{\text {sam }}=d_{\text {ref } 1}+d_{\text {ref } 2}+d_{\text {blood }}
$$




$$
\begin{gathered}
\alpha(f)_{\text {ref } 1}=\frac{1}{d_{\text {ref } 1}} \ln \left(\frac{E_{\text {air }}(f)}{E_{\text {ref } 1}(f)}\right)^{2}, \\
\alpha(f)_{\text {ref } 2}=\frac{1}{d_{\text {ref } 2}} \ln \left(\frac{E_{\text {air }}(f)}{E_{\text {ref } 2}(f)}\right)^{2}, \\
\alpha(f)_{\text {sam }}=\frac{1}{d_{\text {sam }}} \ln \left(\frac{E_{\text {air }}(f)}{E_{\text {sam }}(f)}\right)^{2}, \\
\alpha_{\text {blood }}(f)=\frac{d_{\text {sam }} \alpha_{\text {sam }}(f)-d_{\text {ref } 1} \alpha_{\text {ref } 1}(f)-d_{\text {ref } 2} \alpha_{\text {ref } 2}(f)}{d_{\text {blood }}} .
\end{gathered}
$$

In addition, the blood penetration depth $L_{\text {blood }}$ is the reverse function to the blood absorption coefficient $\alpha_{\text {blood: }}$

$$
L_{\text {blood }}(f)=\frac{1}{\alpha_{\text {blood }}(f)},
$$

The imaginary part of the refractive index $n_{\text {imag blood }}(f)$ requires data about the blood absorption coefficient $\alpha_{\text {blood }}$ :

$$
n_{\text {imagblood }}(f)=\frac{\alpha_{\text {blood }}(f) c}{4 \pi f},
$$

Both parts of the blood permittivity $\varepsilon_{\text {blood }}$ use both parts of the complex refractive index $n_{b l o o d}$ :

$$
\begin{aligned}
& \varepsilon_{\text {real blood }}(f)=n_{\text {real blood }}^{2}(f)-n_{\text {imag blood }}^{2}(f), \\
& \varepsilon_{\text {imag blood }}(f)=2 n_{\text {real blood }}(f) n_{\text {imag blood }}(f) .
\end{aligned}
$$

All of these optical properties are available as results of Spectrina software [21], to obtain the dispersion of optical properties of materials (complex refractive index, complex permittivity, complex conductivity); the spectral characteristics (transmission, reflection, absorption spectra) in transmission and reflection modes.

\section{Transmission experiment results}

Based on 8 biosamples, we have investigated the frequency dispersions of $n_{\text {real }}, \alpha, \varepsilon_{\text {real }}, \varepsilon_{\text {imag }}$ in the THz frequency range (Fig. 5).

After data acquisition, the next step is finding dependencies between glucose concentrations and optical properties. To determine the dependence of blood optical properties upon glucose concentration we selected the experimental data at $0.30,0.350 .40,0.45 \mathrm{THz}$. These frequencies were chosen due to maximal $\mathrm{THz}$ electric field amplitude and minimal absorption of water vapor [28]. Fig. 6 shows the dependences of glucose concentration on the real part of the refractive index and on the real part of the permittivity. The dependence is not linear with blood glucose concentrations above $16 \mathrm{mmol} / \mathrm{l}$. This may be due to the change in physico-chemical properties of blood components with high glucose concentration [29,30]. As known hyperglycemia caused by insulin deficiency is accompanied by a large loss of electrolytes, dehydration of tissues and osmolality increase of blood plasma.

In a week we repeated these experiments to check the type of dependence of glucose level on the blood optical properties. The comparision of data of two experiments is shown in Fig. 6. We observe the shift of the calibration curves but the shape of the curves was similar. This shift may be caused by the variation of the concentrations of the patient blood components (cholesterol, uric acid, etc.) in another experiment.

The dependences of glucose concentration on the real part of the refractive index and the real part of the permittivity were approximated by all OriginPro embedded functions to find the most suitable one. The Gompertz function (formula 12) fitted these dependences better than other ones.

$$
y=A \exp (-\exp (-C(x-B))) .
$$

These dependencies indicate that quantitative blood glucose levels analysis is feasible by using TDS in the $\mathrm{THz}$ frequency range. According to these data, we can simulate glucose level analysis by performing numerical modeling in the reflective mode.

Often, during the process of mixing, analysis the model of effective medium is used. It means the following case: if we mix few media with known optical parameters and proportions, we can calculate optical parameters as the sum of multiplication products of medium concentrations and its optical parameters. In our case, the results 

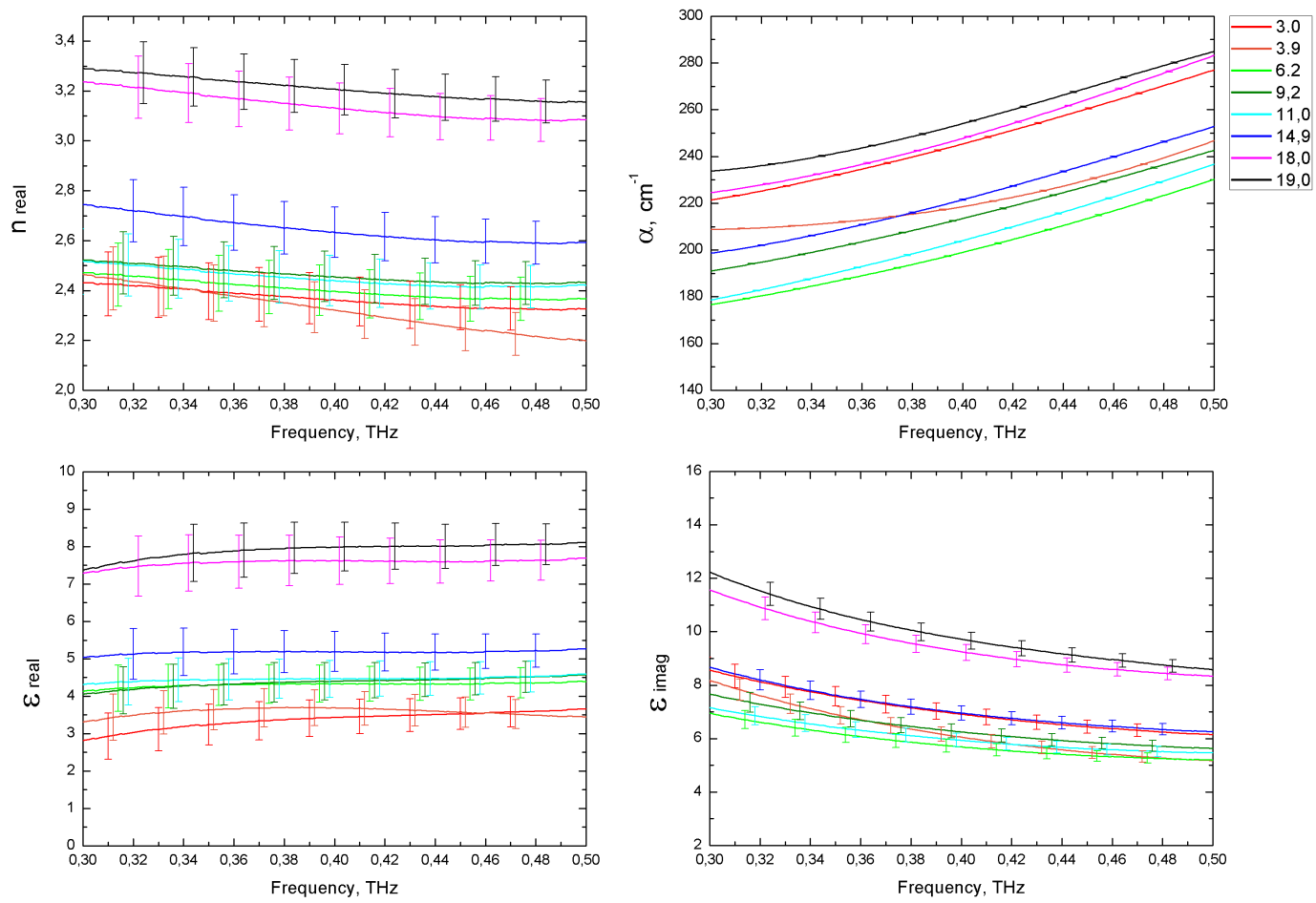

FIG. 5. Dispersions of optical parameters of samples with different glucose concentration $C_{\text {glucose }}$ : subgraph $a$ shows the real part of refractive index $n_{\text {real }}(f), b$ - the absorption coefficient $\alpha(f), c$ - the real part of permittivity $\varepsilon_{\text {real }}(f), d$ - the imaginary part of permittivity $\varepsilon_{\text {imag }}(f)$

TABLE 2. The coefficients of Gompertz fitting for dependence of glucose concentration $C_{g l u c o s e}$ on real parts of refractive index $n_{\text {real }}$ (left tab) and of permittivity $\varepsilon_{\text {real }}$ (right tab) at the frequencies of $0.30,0.35,0.40,0.45 \mathrm{THz}$

\begin{tabular}{|c|c|c|c|c|c|c|}
\hline \multirow{2}{*}{ Frequency } & \multicolumn{2}{|c|}{ Coefficient A } & \multicolumn{2}{c|}{ Coefficient B } & \multicolumn{2}{c|}{ Coefficient C } \\
\cline { 2 - 7 } & Ex.1 & Ex.2 & Ex.1 & Ex.2 & Ex.1 & Ex.2 \\
\hline $0.30 \mathrm{THz}$ & 19.0 & 16.2 & 2.49 & 2.17 & 4.14 & 16.98 \\
\hline $0.35 \mathrm{THz}$ & 19.0 & 16.2 & 2.44 & 2.13 & 4.21 & 14.59 \\
\hline $0.40 \mathrm{THz}$ & 19.0 & 16.2 & 2.40 & 2.12 & 4.24 & 12.71 \\
\hline $0.45 \mathrm{THz}$ & 19.0 & 16.2 & 2.36 & 2.11 & 4.23 & 11.65 \\
\hline
\end{tabular}

\begin{tabular}{|c|c|c|c|c|c|c|}
\hline \multirow{2}{*}{ Frequency } & \multicolumn{2}{|c|}{ Coefficient A } & \multicolumn{2}{c|}{ Coefficient B } & \multicolumn{2}{c|}{ Coefficient C } \\
\cline { 2 - 7 } & Ex.1 & Ex.2 & Ex.1 & Ex.2 & Ex.1 & Ex.2 \\
\hline $0.30 \mathrm{THz}$ & 19.0 & 16.2 & 3.73 & 2.73 & 0.84 & 1.86 \\
\hline $0.35 \mathrm{THz}$ & 19.0 & 16.2 & 3.99 & 3.17 & 0.85 & 4.54 \\
\hline $0.40 \mathrm{THz}$ & 19.0 & 16.2 & 4.07 & 3.21 & 0.87 & 4.30 \\
\hline $0.45 \mathrm{THz}$ & 19.0 & 16.2 & 4.07 & 3.18 & 0.87 & 3.52 \\
\hline
\end{tabular}

cannot be fitted into a model of effective medium theory due to the model being developed for noninteracting components, thus the biochemical interaction of components cannot be fitted into this model [31].

\section{Reflective THz time-domain spectroscopy}

Terahertz radiation is highly absorbed by water and biological tissues, so signal transmission through a biotissue for non-invasive measurements is not possible practically at $\mathrm{THz}$ frequencies. At the same time, it's possible to use $\mathrm{THz}$ time domain spectroscopy in the reflective mode, which allows to study a layered structure [32]. Fig. 7 shows a sketch of $\mathrm{THz}$ spectrometer for this case. The nail may be used as a reference sample for non-invasive glucose level sensing.

The typical reflected $\mathrm{THz}$ waveform obtained at the interaction of $\mathrm{THz}$ pulse with a nail layered structure is shown in Fig. 8.

The time domain of the resulting pulse train detected in the reflection measurements consists of a reference pulse, a pulse reflected from the front of the nail plate, and a sample pulse, a delayed pulse reflected from the nail plate/nail bed interface. The optical properties of the nail bed may be retrieved from the ratio between the sample and reference electric fields obtained experimentally. 

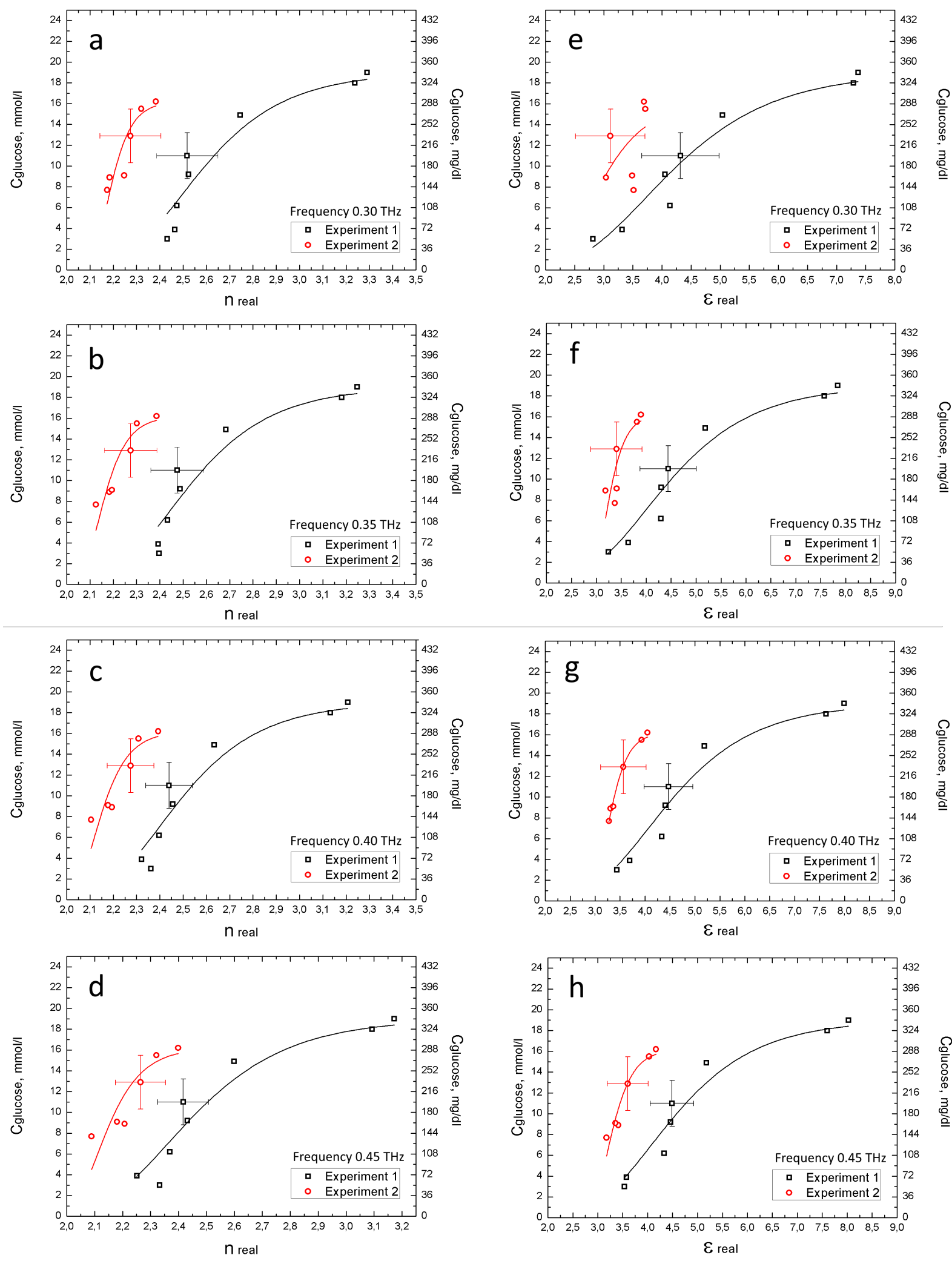

FIG. 6. Dependencies of glucose concentration on the real part of refractive index $C_{\text {glucose }}\left(n_{\text {real }}\right)$ $(\mathrm{a}, \mathrm{b}, \mathrm{c}, \mathrm{d})$ and real part of permittivity $C_{\text {glucose }}\left(\varepsilon_{\text {real }}\right)(\mathrm{e}, \mathrm{f}, \mathrm{g}, \mathrm{h})$ at the frequencies of $0.30,0.35$, $0.40,0.45 \mathrm{THz}$. Gompertz function is used to perform fitting line. Comparison of results of two experiments. 


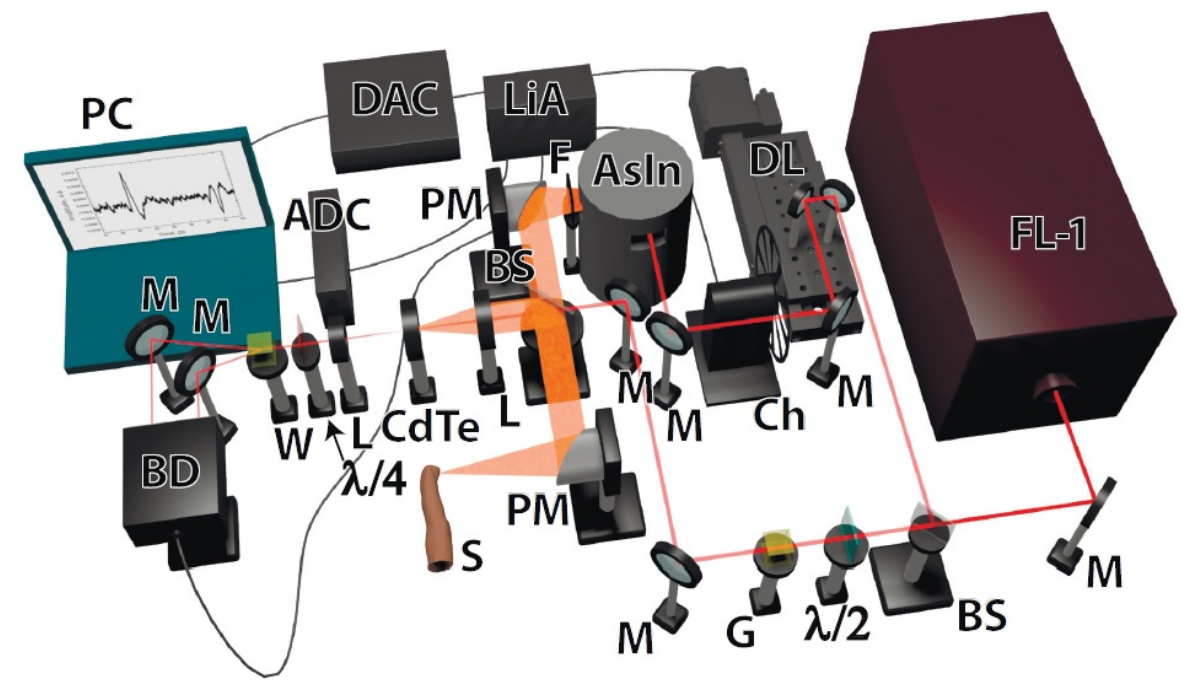

FIG. 7. Sketch of THz TDS spectrometer in reflection mode for glucose level sensing (FL-1 femtosecond laser based on potassium-yttrium tungstate crystal activated with ytterbium (Yb: KYW), generating femtosecond pulses; F - a set of teflon filters for IR wavelength range cutting off, BS - beamsplitter, DL - optical delay line, M - mirrors, S - investigated sample, W Wollaston prism, CdTe - electro-optical cadmium-telluric crystal, BD - balanced detector, LiA lock-in amplifier, PC - personal computer, G - Glan-Taylor prism, PM - parabolic mirrors, Ch chopper, DAC - digital to analog converter, ADC - analog to digital converter)

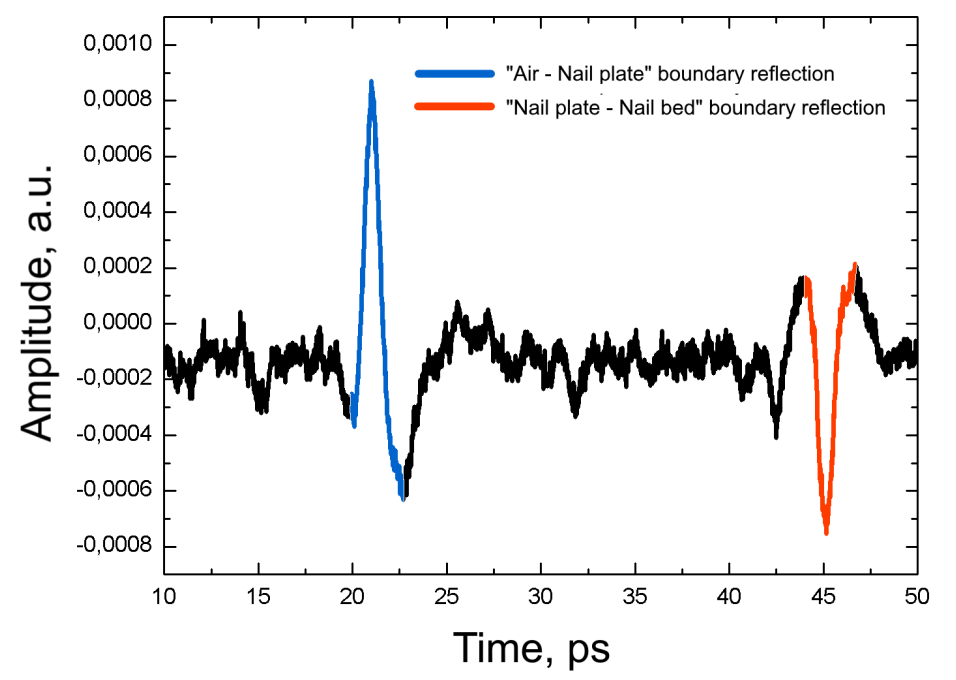

FIG. 8. Typical reflected signal from a human nail

So the nail is a type of layered structure, which has quasi-homogenous layers with defined boundaries: nail plate and nail bed. Moreover, the nail bed contains significant part of capillaries, the optical properties of which strongly depend on the glucose level in blood. Therefore such structure may be simply simulated using the finite difference time domain (FDTD) solver by CST. The simulated nail is shown in Fig. 9.

A waveguide port simulated a TE plane wave propagating through a medium. The port works as an emitter and detector. The THz bipolar waveform of the source was experimentally recorded and was fitted by the formula for a $\mathrm{THz}$ surface field emitter:

$$
E(t)=\frac{2 A}{\tau^{2}} \exp \left(\frac{-(t+\Delta t)^{2}}{\tau^{2}}\right)-4 A \frac{(t+\Delta t)^{2}}{\tau^{4}} \exp \left(\frac{-(t+\Delta t)^{2}}{\tau^{2}}\right),
$$

where $\tau$ is $0.765 \mathrm{ps}, A$ is 0.41 and $\Delta t$ is -5.8 ps. The experimental and theoretical ones are shown in Fig. 10. 


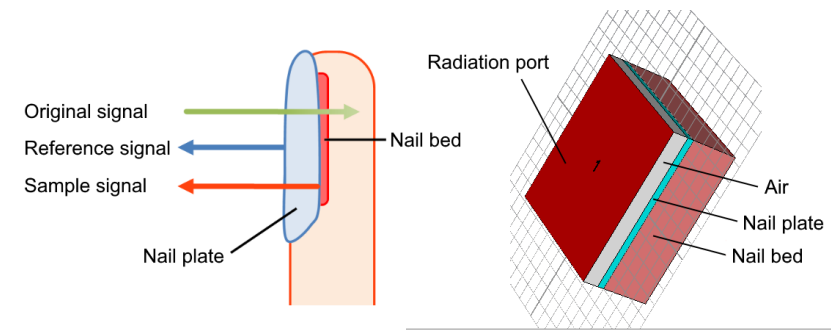

FIG. 9. Principal scheme of the reflecting nail structure (left) and its realization in CST Microwave Studio (right)

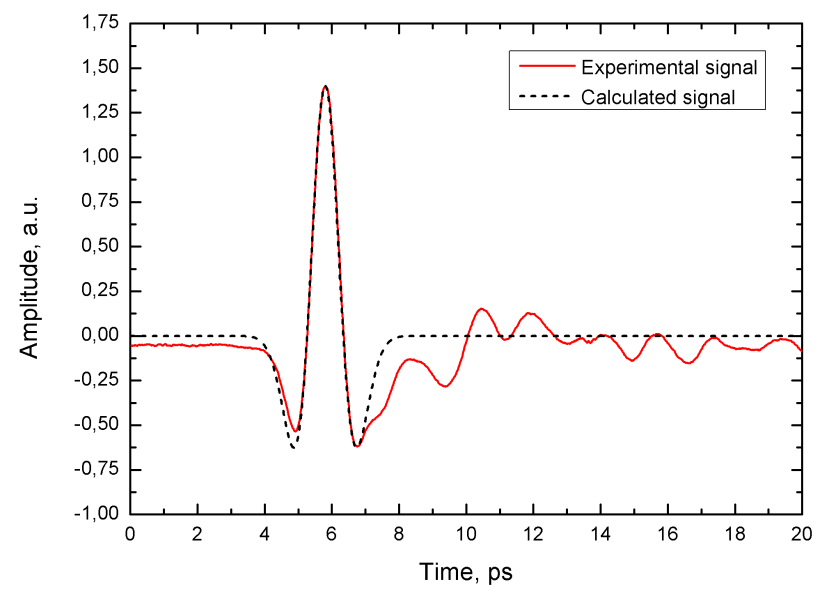

FIG. 10. THz source signal: experimental (solid line) and theoretical (dotted line)

In the model we used the experimental data for the human nails [23] (Fig. 11). The thickness of the nail plate was $0.58 \mathrm{~mm}$.

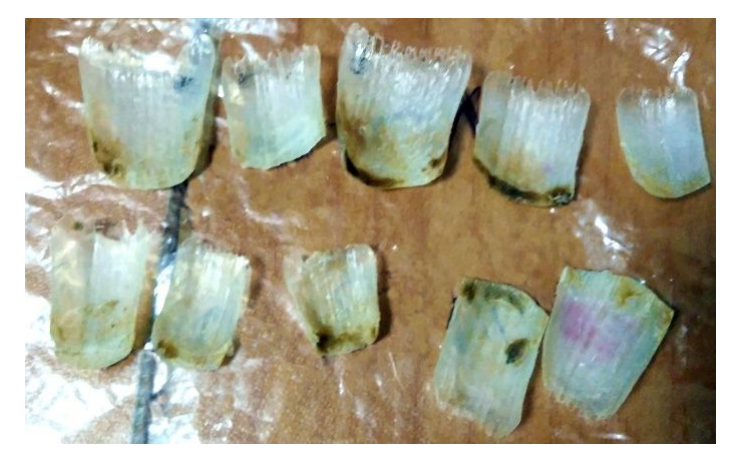

FIG. 11. The nail samples under study

The experimental dispersions of the real and imaginary parts of the nail plate permittivity are shown in Fig. 12 .

The thickness of the nail bed layer was chosen as $3 \mathrm{~mm}$ due to the fact, that we observed only the reflective signal from the structure. It was assumed that the dispersions of nail bed permittivity for the different glucose concentrations can be replaced by the dispersions of the permittivity of blood samples with the different glucose levels. The assumption was made due to the nail bed containing blood-filled capillaries [33] and the main reason the nail bed's optical properties change is due to varying blood glucose concentrations.

Modeled THz waveforms of reflected signals from the nail for different glucose levels are shown in Fig. 13. As seen from the figure, the peak-to-peak amplitude of the sample pulse reflected from the nail plate/nail bed 

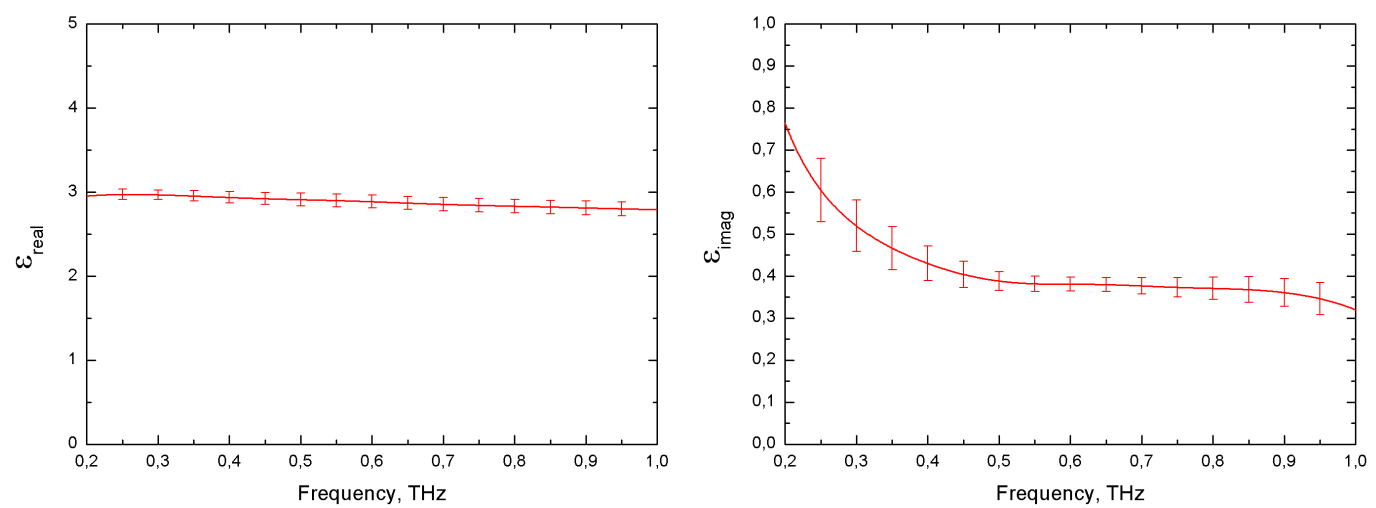

FIG. 12. The dispersion of the real and imaginary parts of the permittivity of the nails. Spectral resolution is $1 \mathrm{GHz}$.

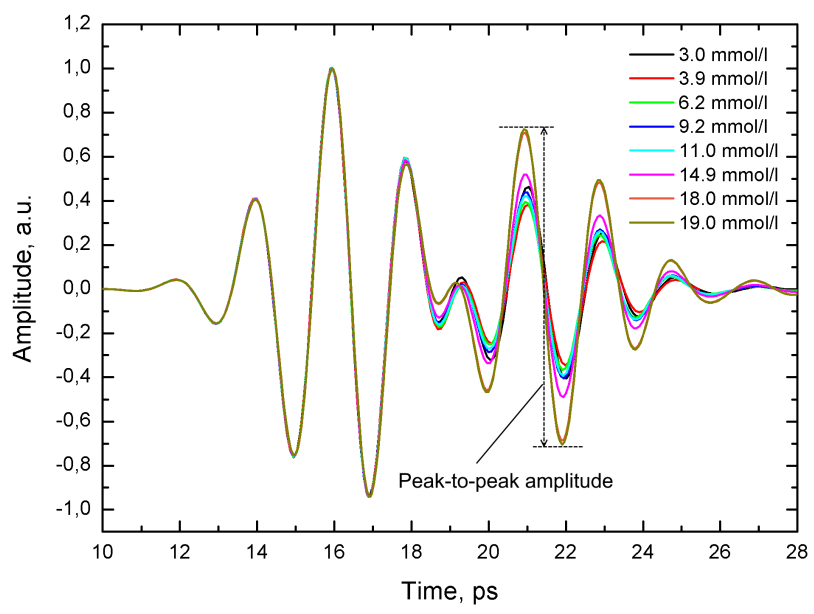

FIG. 13. THz waveforms for the different glucose levels in blood

interface changes with varying glucose levels. The increasing the blood glucose level results in an increase in the amplitude of the pulse reflected from the nail bed (Fig. 14). So the reflection mode of THz TDS may be used as non-invasive technique for glucose level control.

\section{Conclusion}

This work shows the application of THz time domain spectroscopy in the field of glucose sensing. Based on the refractive index dispersion for whole human blood, we demonstrate that different blood glucose levels have individual refractive index dispersions in frequencies ranging from $0.3-0.5 \mathrm{THz}$ and the relationships between the refractive index and glucose concentration at the single $\mathrm{THz}$ frequencies are described by the Gompertz function. Additionally, the dispersion of complex refractive index of human nails in the transmission mode was obtained. Based on these data we proved the possibility of non-invasive glucose level sensing in reflective mode using reflection of $\mathrm{THz}$ pulse from nail plate/nail bed interface. This research may provide a quick, accurate, and continuous method to measure glucose concentration in human blood, which has wide potential application in clinical practice.

\section{Acknowledgements}

This work was financially supported by the Government of Russian Federation, Grant 074-U01. 


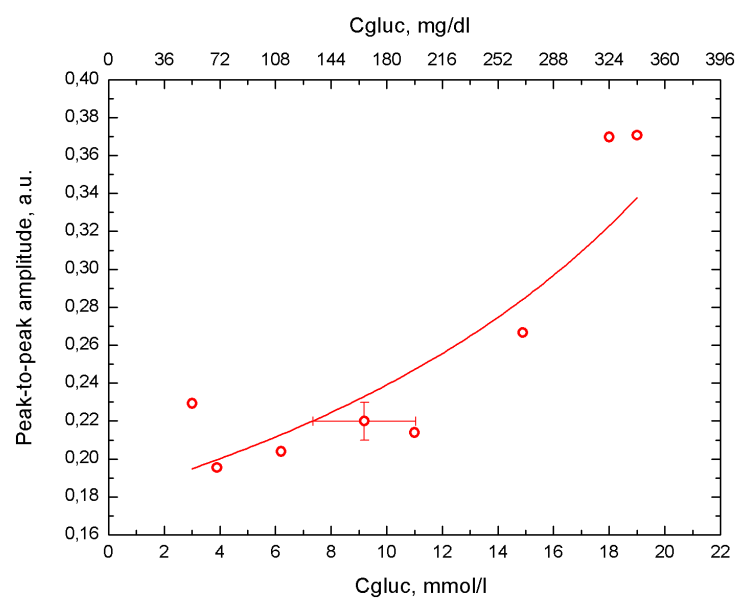

FIG. 14. The dependence of the peak-to-peak amplitude of $\mathrm{THz}$ reflected signal on the glucose level in blood

\section{References}

[1] Shur M. Terahertz technology: devices and applications. Proceedings of ESSDERC, 2005, 35, P. 13-21.

[2] Consolino, L., Bartalini S., and De Natale P. Terahertz Frequency Metrology for Spectroscopic Applications: a Review. Journal of Infrared, Millimeter, and Terahertz Waves, 2017, 38 (11), P. 1289-1315.

[3] Al-Naib, I.,Withawat W. Recent Progress in Terahertz Metasurfaces. Journal of Infrared, Millimeter, and Terahertz Waves, 2017,38 (9), P. $1067-1084$.

[4] Reid C. B., Reese G., Gibson A.P., Wallace V.P. Terahertz time-domain spectroscopy of human blood. IEEE Transactions on Terahertz Science and Technology, 2013, 3 (4), P. 363-367.

[5] Yang X., Zhao X., Yang K., Liu Y., Liu Y., Fu W., Luo Y. Biomedical applications of terahertz spectroscopy and imaging. Trends in biotechnology, 2016, 34 (10), P. 810-824.

[6] Thrane L., Jacobsen R. H., Jepsen P. U., Keiding S. THz reflection spectroscopy of liquid water. Chemical Physics Letters, 1995 , 240 (4), P. 330-333.

[7] Fitzgerald, A. J., Berry, E., Zinovev, N. N., Walker, G.C., Smith, M. A., Chamberlain, J.M. An introduction to medical imaging with coherent terahertz frequency radiation. Physics in Medicine \& Biology, 2002, 47 (7), P. R67.

[8] Wang, K., Da-Wen S., Hongbin P. Emerging non-destructive terahertz spectroscopic imaging technique: Principle and applications in the agri-food industry. Trends in Food Science \& Technology, 2017, 67, P. 93-105.

[9] Stratton I. M., Adler A. I., Neil H. A. W., Matthews D. R., Manley S. E., Cull C. A., Hadden D., Turner R. C., Holman R. R. Association of glycaemia with macrovascular and microvascular complications of type 2 diabetes (UKPDS 35): prospective observational study. British Medical Journal,2000, 321 (7258), P. 405-412.

[10] Zoungas S., Patel A., Chalmers J., de Galan B. E., Li Q., Billot L., Woodward M., Ninomiya T., Neal B., MacMahon S. et al. Severe hypoglycemia and risks of vascular events and death. New England Journal of Medicine, 2010, 363 (15), P. 1410-1418.

[11] Su G., Mi S., Tao H., Li Z., Yang H., Zheng H., Zhou Y., Ma C. Association of glycemic variability and the presence and severity of coronary artery disease in patients with type 2 diabetes. Cardiovascular Diabetology, 2011, 10 (1), P. 19.

[12] Muggeo M., Verlato G., Bonora E., Zoppini G., Corbellini M., De Marco R. Long-term instability of fasting plasma glucose, a novel predictor of cardiovascular mortality in elderly patients with noninsulin-dependent diabetes mellitus: the verona diabetes study. Circulation, 1997, 96 (6), P. 1750-1754.

[13] Krinsley J.S. Glycemic variability: a strong independent predictor of mortality in critically ill patients. Critical Care Medicine, 2008, 36 (11), P. 3008-3013.

[14] Wang X., Zhao X., Dorje T., Yan H., Qian J., Ge J. Glycemic variability predicts cardiovascular complications in acute myocardial infarction patients with type 2 diabetes mellitus. International Journal of Cardiology, 2014, 172 (2), P. 498-500.

[15] Tierney M. J., Tamada J. A., Potts R. O., Jovanovic L., Garg S., Team C. R. et al. Clinical evaluation of the Glucowatch $®$ Biographer: a continual, non-invasive glucose monitor for patients with diabetes. Biosensors and Bioelectronics, 2001, 16 (9-12), P. 621-629.

[16] Larin K. V., Eledrisi M. S., Motamedi M., Esenaliev R. O. Noninvasive blood glucose monitoring with optical coherence tomography: A pilot study in human subjects. Diabetes Care, 2002, 25 (12), P. 2263-2267.

[17] Cherkasova O.P., Nazarov M. M., Smirnova I.N., Angeluts A. A., Shkurinov A.P. Application of time-domain THz spectroscopy for studying blood plasma of rats with experimental diabetes. Physics of Wave Phenomena, 2014, 22 (3), P. 185-188.

[18] Segman Y. New method for computing optical hemodynamic blood pressure. Journal of Clinical and Experimental Cardiology, 2016, 7 (12), P. 1-7.

[19] Chen, Hua, et al. Quantify Glucose Level in Freshly Diabetics Blood by Terahertz Time-Domain Spectroscopy. Journal of Infrared, Millimeter, and Terahertz Waves, 2018, 39 (4), P. 399-408.

[20] Smith J. The Pursuit of Noninvasive Glucose: Hunting the Deceitful. Turkey, 5th Edition. 2017. URL: http://nivglucose.com/The\%20Pursuit $\% 20$ of\%20Noninvasive\%20Glucose $\% 205$ th\%20Edition.pdf. 
[21] Gusev S. I., Borovkova M. A., Strepitov M. A., Khodzitsky M. K. Blood optical properties at various glucose level values in THz frequency range. Proceedings of the SPIE-OSA, 2015, 9537, P. 95372.

[22] Gusev S. I., Balbekin N.S., Sedykh E. A., Kononova Yu. A., Litvinenko E. V., Goryachuk A.A., Begaeva V. A., Goryachuk A. A., Babenko A. Yu., Khodzitsky M.K. Influence of creatinine and triglycerides concentrations on blood optical properties of diabetics in THz frequency range. Journal of Physics: Conference Series, 2016, 735 (1), P. 012088.

[23] Guseva V. A., Gusev S. I., Demchenko P. S., Sedykh E. A., Khodzitsky M. K. Optical properties of human nails in THz frequency range. Journal of Biomedical Photonics \& Engineering, 2016, 2 (4), P. 040306.

[24] Bespalov V. G., Gorodetskiĭ A. A., Denisyuk I. Yu., Kozlov S. A., Krylov V. N., Lukomskiĭ G. V., Petrov N. V., Putilin S. E. Methods of generating superbroadband terahertz pulses with femtosecond lasers. Journal of Optical Technology, 2008, 75 (10), P. 636-642.

[25] Zhang, Xi-Cheng, Jingzhou Xu. Introduction to THz wave photonics. Springer, New York, 2010., P. 40-43.

[26] Sheffield C.A., Kane M.P., Bakst G., Busch R. S., Abelseth J. M., Hamilton R. A. Accuracy and precision of four value-added blood glucose meters: the Abbott Optium, the DDI Prodigy, the HDI True Track, and the HypoGuard Assure Pro. Diabetes Technology \& Therapeutics, 2009, 11 (9), P. 587-592.

[27] Biester T., Danne T., Bläsig S., Remus K., Aschemeier B., Kordonouri O., Bardtrum L., Haahr H. Pharmacokinetic and prandial pharmacodynamic properties of insulin degludec/insulin aspart in children, adolescents, and adults with type 1 diabetes. Pediatric Diabetes, 2016, 17 (8), P. 642-649.

[28] Van Exter M., Fattinger C., Grischkowsky D. Terahertz timedomain spectroscopy of water vapor. Optics letters, 1989, 14 (20), P. 11281130.

[29] Bogner P., Sipos K., Ludany A., Somogyi B., Miseta A. Steadystate volumes and metabolism-independent osmotic adaptation in mammalian erythrocytes. European Biophysics Journal, 2002, 31 (2), P. 145-152.

[30] Jain S. K. Hyperglycemia can cause membrane lipid peroxidation and osmotic fragility in human red blood cells. Journal of Biological Chemistry, 1989, 264 (35), P. 21340-21345.

[31] Son J.-H. Terahertz Biomedical Science and Technology. CRC Press, Boca Raton., 2014. P. 347-350.

[32] Němec H., Kadlec F., Kužel P, Duvillaret L, Coutaz L. Independent determination of the complex refractive index and wave impedance by time-domain terahertz spectroscopy. Optics communications, 2006, 260 (1), P. 175-183.

[33] Hasegawa K., Pereira B.P. The microvasculature of the nail bed, nail matrix, and nail fold of a normal human fingertip. Journal of Hand Surgery, 2001, 26 (2), P. 283-290. 